Claremont Colleges

Scholarship@ Claremont

All HMC Faculty Publications and Research

HMC Faculty Scholarship

$12-22-2008$

\title{
Constructing Diabatic States from Adiabatic States: Extending Generalized Mulliken-Hush to Multiple Charge Centers with Boys Localization
}

Joseph E. Subotnik

Tel Aviv University; Northwestern University

Robert J. Cave

Harvey Mudd College

Sina Yeganeh

Northwestern University

Mark A. Ratner

Northwestern University

\section{Recommended Citation}

Constructing diabatic states from adiabatic states: Extending generalized Mulliken--Hush to multiple charge centers with Boys localization. Joseph E. Subotnik, Sina Yeganeh, Robert J. Cave, and Mark A. Ratner, J. Chem. Phys. 129, 244101 (2008), DOI:10.1063/1.3042233. in All HMC Faculty Publications and Research by an authorized administrator of Scholarship @ Claremont. For more information, please contact scholarship@cuc.claremont.edu. 


\title{
Constructing diabatic states from adiabatic states: Extending generalized Mulliken-Hush to multiple charge centers with Boys localization
}

\author{
Joseph E. Subotnik, ${ }^{1,2, a)}$ Sina Yeganeh, ${ }^{2}$ Robert J. Cave, ${ }^{3}$ and Mark A. Ratner ${ }^{2}$ \\ ${ }^{1}$ School of Chemistry, Tel-Aviv University, Tel-Aviv 69978, Israel \\ ${ }^{2}$ Department of Chemistry, Northwestern University, 2145 Sheridan Road, Evanston, Illinois 60208, USA \\ ${ }^{3}$ Department of Chemistry, Harvey Mudd College, Claremont, California 91711, USA
}

(Received 25 September 2008; accepted 16 November 2008; published online 22 December 2008)

\begin{abstract}
This article shows that, although Boys localization is usually applied to single-electron orbitals, the Boys method itself can be applied to many electron molecular states. For the two-state charge-transfer problem, we show analytically that Boys localization yields the same charge-localized diabatic states as those found by generalized Mulliken-Hush theory. We suggest that for future work in electron transfer, where systems have more than two charge centers, one may benefit by using a variant of Boys localization to construct diabatic potential energy surfaces and extract electronic coupling matrix elements. We discuss two chemical examples of Boys localization and propose a generalization of the Boys algorithm for creating diabatic states with localized spin density that should be useful for Dexter triplet-triplet energy transfer. (C) 2008 American Institute of Physics. [DOI: 10.1063/1.3042233]
\end{abstract}

\section{INTRODUCTION}

\section{A. Diabatic surfaces}

The construction of diabatic potential energy surfaces from adiabatic surfaces is a crucial step when modeling electron transfer. In particular, one needs two diabatic states to represent the initial and final states of the electron transfer process. Mathematically, diabatic states are usually defined as those states for which the derivative couplings $\left(\left\langle\Phi_{i}(r ; R)\left|\nabla_{R}\right| \Phi_{j}(r ; R)\right\rangle_{r}\right)$ would be equal to zero. ${ }^{1-3}$ Here, $\left|\Phi_{i}(r ; R)\right\rangle$ and $\left|\Phi_{j}(r ; R)\right\rangle$ are eigenvectors of the electronic Hamiltonian and \langle\rangle$_{r}$ signifies integration over all electronic degrees of freedom. Because exactly diabatic states do not usually exist (as shown by Mead and Truhlar ${ }^{4}$ ), effectively one has a great deal of flexibility in choosing a definition for quasidiabatic (or just "diabatic") states. For a good overview, see Refs. 5-7. Over the last few decades, several research groups have proposed different prescriptions for constructing diabatic states, each with different advantages and disadvantages and with different domains of applicability. Our interest is in designing diabatic states which are relevant to electron transfer.

Broadly speaking, current approaches for constructing diabatic surfaces can be divided into three categories. We will now describe a few of these methods, but we refer the reader to Ref. 5 for a much more thorough analysis. The first approach toward diabatization is to calculate the derivative couplings in the adiabatic basis, and then define diabatic states as those rotated states with near zero derivative couplings. Baer and co-workers ${ }^{3,8,9}$ provided an algorithm for finding these states. We note, however, that computing derivative coupling elements can be very expensive and this

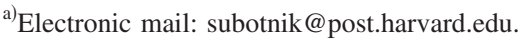

method is usually not applied to molecules with more than a few atoms. An active area of research is finding ways to compute derivative couplings more easily. ${ }^{10}$

A second approach toward diabatization is to avoid calculating derivative coupling elements explicitly, but rather minimize them implicitly by designing diabatic states with intuitively desirable mathematical characteristics. Popular examples of these approaches are block diagonalization ${ }^{11,12}$ and the fourfold way ${ }^{13-17}$ (see also Ref. 18). As conceived by Pacher et al., ${ }^{11,12}$ the block diagaonalization algorithm rotates together a small set of adiabatic states using the unique transformation that minimizes the distance (in wave-function space) between the target diabatic states and a reference basis of nearly diabatic states (which is assumed always available). Block diagonalization was implemented within a CASSCF framework by Domcke et al. ${ }^{19,20}$

Instead of relying on reference states (as in block diagonalization), Ruedenberg and Atchity ${ }^{16,17}$ investigated the configuration interaction expansions for a group of adiabatic states in a well-defined basis of single-electron diabatic molecular orbitals (to be constructed). The authors then defined diabatic states roughly as those states for which the dominant configurations were unchanged over the entire potential energy surface, thus introducing a notion of "configurational uniformity." The fourfold way approach of Nakamura and Truhlar $^{13-15}$ extended the ideas of Atchity and Ruedenberg by generalizing and stabilizing the method for constructing diabatic orbitals, introducing the notion of "molecular orbital uniformity." Up until now, the fourfold way has usually been applied to systems with under ten atoms.

A third approach for constructing diabatic potential energy surfaces is to invoke a physical observable, often the dipole operator, in order to characterize diabatic states without worrying about the details of any particular electronic structure method. Of these methods, the most common is the 
generalized Mulliken-Hush (GMH) algorithm by Cave and Newton, ${ }^{21,22}$ which was designed for use in electron transfer calculations. The GMH algorithm uses physical intuition to construct diabatic states as the initial and final states in an electron transfer event based on charge separation along a central direction. While the GMH algorithm has several drawbacks, most especially its assumption of a central charge-transfer direction, ${ }^{23}$ the strength of the GMH algorithm is its computational speed and applicability to large electron transfer calculations. Electron transfer systems are large and the calculations can be computationally demanding. Most often theorists have preferred to avoid calculating diabatic states on the basis of derivative couplings or configurational uniformity, choosing instead to extract electronic couplings using approximate methods or perturbation theory. ${ }^{24-31}$ Our central objective in this paper is to extend the GMH formalism with Boys localization, so that we can both construct rigorous diabatic states when there are many charge centers and also extract electronic coupling elements for large electron transfer calculations.

\section{B. Generalized Mulliken-Hush theory}

The general idea behind GMH theory is to recognize that, in electron transfer, the diabatic states should correspond to charge localized on different centers (donors and acceptors). More specifically, according to standard GMH theory, one constructs diabatic states as follows:

(1) One calculates all dipole matrix elements of the adiabatic states.

(2) For the two-state problem, one recognizes that the important direction is the direction of the dipole moment of the initial adiabatic state minus the dipole moment of the final adiabatic state: $\vec{v}_{0}=\left(\vec{\mu}_{11}-\vec{\mu}_{22}\right) /\left|\vec{\mu}_{11}-\vec{\mu}_{22}\right|$. When there are more than two states, GMH theory suggests that one construct $v_{0}$ as the average over several different initial and final states. One projects all dipole matrix elements into the $v_{0}$ direction, and then one diagonalizes the dipole matrix. For the two-state case, the rotation matrix that comes out of the diagonalization is the GMH transformation matrix from adiabatic to diabatic states.

(3) For the multistate case, one must consider that several states may have charge on the same center. In that case, one diagonalizes the Hamiltonian for each same-center block. The resulting states are the locally adiabatic GMH diabatic states.

For molecular configurations with two or more charge centers in a linear or near-linear geometry, GMH theory works very well at constructing diabatic states. Its generalization to three or more charge centers in a noncollinear geometry is less satisfying, however, because it does not localize charge very well for bent systems. The source of the problem is that, for bent systems, there is no unique vector $v_{0}$ to project dipoles into: the averaging in step No. 2 above is not detailed enough.

\section{Boys localization}

We turn our attention to the discipline of electronic structure theory (EST) where the subject of electronic localization is also encountered, but with a very different purpose. For localization in EST, one begins with the canonical orbitals $\left\{\phi_{i}\right\}$ from a Hartree-Fock calculation and one generates localized orbitals $\left\{\eta_{i}\right\}$ for two reasons: (i) chemical intuition $^{32}$ and (ii) computational speed-ups when doing local correlation theory. ${ }^{33}$ Mathematically, localized orbitals are constructed as follows: ${ }^{34-38}$ We begin with canonical orbitals $\left\{\phi_{1}, \ldots, \phi_{n}\right\}$ and we introduce a rotation matrix $\mathbf{U}$ $\left(\mathbf{U U}^{T}=\mathbf{I d}\right)$, which mixes the canonical orbitals:

$$
\eta_{i}=\sum_{j} \phi_{j} U_{j i}, \quad i=1, \ldots, n .
$$

We force the new orbitals $\left\{\eta_{1}, \ldots, \eta_{n}\right\}$ to be localized by insisting that they maximize a localization function. For Boys localization, ${ }^{35,36,39}$ we insist the localized orbitals $\left\{\eta_{i}\right\}$ maximize

$$
f_{\text {Boys }}(\mathbf{U})=f_{\text {Boys }}\left(\left\{\eta_{i}\right\}\right)=\sum_{i, j}\left|\left\langle\eta_{i}|\mathbf{R}| \eta_{i}\right\rangle-\left\langle\eta_{j}|\mathbf{R}| \eta_{j}\right\rangle\right|^{2} .
$$

Note that the localization function $f_{\text {Boys }}$ is a quartic function of the rotational matrix $U$ in Eq. (1). If we take the derivative of Eq. (2) with respect to $\mathbf{U}$, we find that, for Boys localization, the localized orbitals satisfy ${ }^{40}$

$$
\left(\left\langle\eta_{i}|\mathbf{R}| \eta_{i}\right\rangle-\left\langle\eta_{j}|\mathbf{R}| \eta_{j}\right\rangle\right) \cdot\left\langle\eta_{i}|\mathbf{R}| \eta_{j}\right\rangle=0, \quad i, j=1, \ldots, n .
$$

In practice, for molecules in their ground-state equilibrium geometry, the Boys localized orbitals are usually either bonding orbitals (centered between atoms) or lone-pair orbitals (centered around atoms). It is known that Boys localized orbitals do not preserve $\sigma-\pi$ symmetry (resulting, e.g., in "banana bonds" for benzene ${ }^{41}$ ). This is discussed more in Sec. III.

\section{The connection between GMH and Boys localization}

The Boys approach can be extended beyond orbital localization to state localization. In so doing, one can prove that under certain approximations, the Boys solution is equivalent to the GMH result. We demonstrate this now.

The application of Boys localization to adiabatic states (rather than orbitals) is straightforward. Given $N_{s}$ adiabatic states, $\left\{\Phi_{i}\right\}$, in analogy to Eq. (1), we may construct a diabatic state representation $\left\{\Xi_{i}\right\}$, as a function of a rotation matrix $\mathbf{U}$ :

$$
\Xi_{i}=\sum_{j=1}^{N_{s}} \Phi_{j} U_{j i}, \quad i=1, \ldots, N_{s} .
$$

In analogy to Eq. (2), we define the Boys localized diabatic states to be those states which move the charge centers as far away from each other as possible, maximizing the localization sum: 


$$
f_{\text {Boys }}(\mathbf{U})=f_{\text {Boys }}\left(\left\{\Xi_{i}\right\}\right)=\sum_{i, j=1}^{N_{s}}\left|\left\langle\Xi_{i}|\vec{\mu}| \Xi_{i}\right\rangle-\left\langle\Xi_{j}|\vec{\mu}| \Xi_{j}\right\rangle\right|^{2} .
$$

In Sec. II, we will show analytically that the GMH approach for two-state problems is an approximation to the Boys localization of electronic states. Afterwards, we will employ Boys localization to localize charge-transfer states for (i) benzene surrounded by three $p$-benzoquinone molecules and (ii) a square of $\mathrm{He}$ atoms with a net positive charge, $\mathrm{He}_{4}^{+}$. These chemical examples demonstrate how Boys localization extends the GMH model to charge-transfer systems with more than two charge centers. In the future, we believe that Boys localization will be a useful tool for computational chemists calculating coupling elements and rates of electron transfer.

\section{MIXING TWO ADIABATIC STATES}

We now show that, for the two-state system, the GMH formalism $^{21,22}$ is an approximation derivable from the Boys localization formalism. ${ }^{35,36,38,39}$

\section{A. A quick derivation of the GMH result}

Suppose that, using standard techniques from quantum chemistry, we have generated two adiabatic states $\left(\Phi_{1}, \Phi_{2}\right)$ with dipole matrix elements $\vec{\mu}_{11}, \vec{\mu}_{22}$, and $\vec{\mu}_{12}$. The GMH formalism defines diabatic states $\left(\Xi_{A}, \Xi_{B}\right)$ by rotating the adiabatic states through an angle $\theta$ :

$$
\begin{aligned}
& \Xi_{A}=\cos \theta \Phi_{1}+\sin \theta \Phi_{2}, \\
& \Xi_{B}=-\sin \theta \Phi_{1}+\cos \theta \Phi_{2} .
\end{aligned}
$$

The dipole matrix elements for these new diabatic states are

$$
\begin{aligned}
\vec{\mu}_{A A} & =\left\langle\Xi_{A}|\vec{\mu}| \Xi_{A}\right\rangle \\
& =\cos ^{2} \theta \vec{\mu}_{11}+\sin ^{2} \theta \vec{\mu}_{22}+2 \cos \theta \sin \theta \vec{\mu}_{12}, \\
\vec{\mu}_{B B} & =\left\langle\Xi_{B}|\vec{\mu}| \Xi_{B}\right\rangle \\
& =\sin ^{2} \theta \vec{\mu}_{11}+\cos ^{2} \theta \vec{\mu}_{22}-2 \cos \theta \sin \theta \vec{\mu}_{12}, \\
\vec{\mu}_{A B} & =\left\langle\Xi_{A}|\vec{\mu}| \Xi_{B}\right\rangle \\
& =\left(\cos ^{2} \theta-\sin ^{2} \theta\right) \vec{\mu}_{12}-\cos \theta \sin \theta\left(\vec{\mu}_{11}-\vec{\mu}_{22}\right) \\
& =\cos 2 \theta \vec{\mu}_{12}-\frac{1}{2} \sin 2 \theta\left(\vec{\mu}_{11}-\vec{\mu}_{22}\right) .
\end{aligned}
$$

Because the direction of interest is assumed to be along $\vec{\mu}_{11}-\vec{\mu}_{22}$ the next step replaces all dipole elements in Eqs. (8)-(14) with their projections in the $\vec{v}_{0}$ direction (where $\vec{v}_{0}$ $=\left(\vec{\mu}_{11}-\vec{\mu}_{22}\right) /\left|\vec{\mu}_{11}-\vec{\mu}_{22}\right|$ and the projections are denoted below by a superscript $v$ ). When we project Eq. (14) into the $\vec{v}_{0}$ direction, we get

$$
\mu_{A B}^{v}=\cos 2 \theta \mu_{12}^{v}-\frac{1}{2} \sin 2 \theta\left|\vec{\mu}_{11}-\vec{\mu}_{22}\right| .
$$

Finally, one defines the GMH states as those diabatic states satisfying $\mu_{A B}^{v}=0$. This requires

$$
\begin{aligned}
& \tan 2 \theta=\frac{2 \mu_{12}^{v}}{\left|\vec{\mu}_{11}-\vec{\mu}_{22}\right|}, \\
& \sin 2 \theta=\frac{2 \mu_{12}^{v}}{\sqrt{\left|\vec{\mu}_{11}-\vec{\mu}_{22}\right|^{2}+4\left(\mu_{12}^{v}\right)^{2}}} .
\end{aligned}
$$

This implies that

$$
\begin{aligned}
\left|H_{A B}\right| & =\left|\left\langle\Xi_{A}|\vec{\mu}| \Xi_{B}\right\rangle\right| \\
& =\left|\cos 2 \theta H_{12}-\frac{1}{2} \sin 2 \theta\left(H_{11}-H_{22}\right)\right|, \\
\left|H_{A B}\right| & =\frac{\left|\mu_{12}^{v}\right|\left|H_{11}-H_{22}\right|}{\sqrt{\left|\vec{\mu}_{11}-\vec{\mu}_{22}\right|^{2}+4\left(\mu_{12}^{v}\right)^{2}}} .
\end{aligned}
$$

Here, we have set $H_{12}=0$ because we are working in an adiabatic basis.

Equation (20) is the exact GMH formula for the coupling element, $H_{A B}$. For linear systems, one often approximates that $\vec{\mu}_{12}$ is along the direction of $\vec{\mu}_{11}-\vec{\mu}_{22}$, and one may then replace

$$
\left|\mu_{12}^{v}\right|^{2} \approx\left|\vec{\mu}_{12}\right|^{2} \text {. }
$$

This leads to an approximate GMH result that is often used in calculations:

$$
\left|H_{A B}\right|=\frac{\left|\mu_{12}\right|\left|H_{11}-H_{22}\right|}{\sqrt{\left|\vec{\mu}_{11}-\vec{\mu}_{22}\right|^{2}+4\left|\mu_{12}\right|^{2}}} .
$$

\section{B. Boys localization according to $2 \times 2$ Jacobi sweeps}

We will now derive the same result from Boys localization. Boys localization is defined by Eq. (5) above, which is to be maximized by rotating all of the relevant states. Edmiston and Ruedenberg ${ }^{35}$ decided to maximize functions such as Eq. (5), which are quartic in the rotation matrix, by doing so-called two-by-two "Jacobi sweeps" over pairs of states. As Edmiston and Ruedenberg showed, for the case of two states, such functions can be maximized exactly. Moreover, in order to maximize the function globally for many states, which can be spread out over arbitrarily many charge centers, one can iteratively maximize over all pairs of orbitals until total convergence. Although Edmiston and Ruedenberg initially worked on a different localizing function, Kleier $e t$ $a l .{ }^{38}$ showed the direct application of this approach to Boys localization. When using Jacobi sweeps, maximizing the Boys function is usually very rapid, regardless of the number of charge centers.

For the two-state problem, the solution for the angle $\theta$ [defined in Eq. (6)] can be written most compactly as follows: ${ }^{35,38}$

$$
\begin{aligned}
F & =\left|\vec{\mu}_{12}\right|^{2}-\frac{1}{4}\left|\vec{\mu}_{11}\right|^{2}-\frac{1}{4}\left|\vec{\mu}_{22}\right|^{2}+\frac{1}{2} \vec{\mu}_{11} \cdot \vec{\mu}_{22} \\
& =\left|\vec{\mu}_{12}\right|^{2}-\frac{\left|\vec{\mu}_{11}-\vec{\mu}_{22}\right|^{2}}{4},
\end{aligned}
$$




$$
\begin{aligned}
& G=\vec{\mu}_{12} \cdot\left(\vec{\mu}_{11}-\vec{\mu}_{22}\right), \\
& \cos 4 \theta=\frac{-F}{\left(F^{2}+G^{2}\right)^{1 / 2}} .
\end{aligned}
$$

This defines the diabatic states using the Boys formalism. We may now compute the new Hamiltonian matrix element that couples diabatic states $A$ and $B$ (again using the fact that $H_{12}=0$ since $\Phi_{1}$ and $\Phi_{2}$ are adiabatic states):

$$
\begin{aligned}
\left|H_{A B}\right| & =\left|\left\langle\Xi_{A}|H| \Xi_{B}\right\rangle\right| \\
& =\left|\left(\cos ^{2} \theta-\sin ^{2} \theta\right) H_{12}-\cos \theta \sin \theta\left(H_{11}-H_{22}\right)\right| \\
& =\frac{1}{2}|\sin 2 \theta|\left|H_{11}-H_{22}\right| \\
& =\frac{1}{2 \sqrt{2}} \sqrt{1-\cos 4 \theta}\left|H_{11}-H_{22}\right| \\
& =\frac{1}{2 \sqrt{2}} \sqrt{1+\frac{F}{\left(F^{2}+G^{2}\right)^{1 / 2}}\left|H_{11}-H_{22}\right|} \\
& =\frac{1}{2 \sqrt{2}} \sqrt{1+\frac{\left|\vec{\mu}_{12}\right|^{2}-\frac{\left|\vec{\mu}_{11}-\vec{\mu}_{22}\right|^{2}}{4}}{\left(\left(\left|\vec{\mu}_{12}\right|^{2}-\frac{\left|\vec{\mu}_{11}-\vec{\mu}_{22}\right|^{2}}{4}\right)^{2}+\left(\vec{\mu}_{12} \cdot\left(\vec{\mu}_{11}-\vec{\mu}_{22}\right)\right)^{2}\right)^{1 / 2}}\left|H_{11}-H_{22}\right| .}
\end{aligned}
$$

Equation (32) is the exact coupling element of the diabatic states constructed by Boys localization. This messy expression can be simplified, however, if we invoke the GMH approximation that the only important direction is along $\vec{v}_{0}$ $=\left(\vec{\mu}_{11}-\vec{\mu}_{22}\right) /\left|\vec{\mu}_{11}-\vec{\mu}_{22}\right|$. More specifically, if we assume, as in Eq. (21) that $\vec{\mu}_{12}^{v} \approx \vec{\mu}_{12}$, then the equations simplify greatly.

It follows then that

$$
\begin{aligned}
& G^{2}=\left|\vec{\mu}_{12} \cdot\left(\vec{\mu}_{11}-\vec{\mu}_{22}\right)\right|^{2} \\
& \approx\left|\vec{\mu}_{12}^{v}\right|^{2}\left|\vec{\mu}_{11}-\vec{\mu}_{22}\right|^{2} \\
&=\left|\vec{\mu}_{12}\right|^{2}\left|\vec{\mu}_{11}-\vec{\mu}_{22}\right|^{2}, \\
&\left(F^{2}+G^{2}\right)^{1 / 2}=\left|\vec{\mu}_{12}\right|^{2}+\frac{\left|\vec{\mu}_{11}-\vec{\mu}_{22}\right|^{2}}{4}, \\
&\left|H_{A B}\right| \approx \frac{\left|\vec{\mu}_{12}\right|\left|H_{11}-H_{22}\right|}{\sqrt{\left|\vec{\mu}_{11}-\vec{\mu}_{22}\right|^{2}+4\left|\vec{\mu}_{12}\right|^{2}}}
\end{aligned}
$$

The information thrown away in going from Eq. (32) to Eq. (37) comes from the different possible geometries and positioning of the acceptor and the donor. When we can safely approximate that $\vec{\mu}_{12}$ and $\vec{\mu}_{11}-\vec{\mu}_{22}$ are in the same direction, there is an equivalence between GMH diabatization and Boys localization for the two-state system. This should be the case for small systems with two charge centers, such as $\mathrm{Zn}_{2} \mathrm{H}_{2} \mathrm{O}^{+}$, considered earlier by Cave and Newton, ${ }^{21,22}$ as well as other charge-transfer systems with an obvious and dominating charge-transfer direction. Where there is cylindrical symmetry going from the donor to acceptor, GMH and Boys give exactly the same coupling element. However, for more complicated systems without symmetry, there must be differences between the Boys and GMH approaches, even for two-state systems. This will be investigated in a future paper. ${ }^{42}$

\section{DISCUSSION}

\section{A. Extension to multiple charge centers and caveats}

Unlike the GMH algorithm, the procedure for constructing Boys localized diabatic states can be naturally extended to arbitrarily many diabatic states and charge centers which are in a noncollinear geometry. Mathematically, the fundamental difference between GMH theory and Boys localization is that Boys localization is a quartic function of the rotation mixing states, while GMH theory is quadratic. As written down more precisely above, Boys localization is defined by

$$
\vec{\mu}_{A B} \cdot\left(\vec{\mu}_{A A}-\vec{\mu}_{B B}\right)=0,
$$

while the GMH formalism is defined (roughly speaking) by

$$
\vec{\mu}_{A B} \cdot\left(\vec{\mu}_{11}-\vec{\mu}_{22}\right)=0 .
$$

Thus, GMH is easier to solve-it requires only diagonalization-while Boys localized states must be solved iteratively. The advantage of Boys localization, however, is that Boys localized states can treat charge localized on multiple centers in any geometric configuration. Moreover, like 
GMH theory, the Boys algorithm requires only dipole matrix elements and can be solved with lightning speed relative to the prerequisite electronic structure calculations needed to calculate the dipole matrix elements. To find the Boys localized states, as originally prescribed by Edmiston and Ruedenberg and described thoroughly in Ref. 38, one maximizes the localization function over all pairs of orbitals, iterating until convergence. The nuts and bolts of this "Jacobi sweeps" algorithm have been implemented in most standard quantum-chemistry packages, including Q-CHEM. $^{43}$ Thus, there are few obstacles preventing the use of Boys localization to calculate charge-transfer coupling $H_{A B}$ for complicated molecular geometries with noncollinear charge centers.

We now mention, however, one big difference between Boys localized orbitals and Boys localized states. For Boys localized orbitals, one always wants as much localization as possible because single-electron density describes bonds and lone pairs, and these quantities ought to be locally distributed around atoms. For Boys localized states, however, we want electronic configurations that represent asymptotic limits of electron transfer. This does not translate into as much localization as possible. Rather, a diabatic charge-transfer state should keep the Hamiltonian as diagonal as possible while maintaining the charge-transfer quality of the wavefunction. Thus, for cases where we have more diabatic states than charge centers, we should isolate all diabatic states with charge localized on the same center, and then diagonalize the Hamiltonian sub-block for these states (just as for GMH theory). Of course, for cases where the number of adiabatic states is equal to the number of charge centers, we should find that the Boys localized diabatic states localize charge as physically as possible, one state per charge center. This completes the recipe for how Boys localization can be applied to adiabatic states.

In a future paper, ${ }^{42}$ we will thoroughly investigate the quality of diabatic states produced by this Boys localization routine for electron transfer. While we can be confident that the Boys routine will generate localized diabatic states, it is possible that the routine will localize charge too efficiently, breaking important symmetries along the way. If so, it may be fruitful to consider another popular routine in quantum chemistry for localizing single-electron orbitals that can be applied to localizing states, namely, the Pipek-Mezey (PM) approach ${ }^{37}$ For orbitals, the PM approach is based on maximizing the square of the Mulliken populations on each atom. ${ }^{44}$ For the single-electron picture, Boys orbitals and PM orbitals are usually similar, but PM orbitals usually preserve $\sigma-\pi$ separation, while Boys orbitals do not. The PM approach may not be easily extended to $n$-electron states because of the nonorthogonal atomic basis, but if we consider Lowdin populations instead of Mulliken populations, then the approach can certainly be extended to $n$-electron states. We note that the Boys and PM functions are both quartic functions of the rotation matrix. We wonder what, if any, will be the differences between these algorithms when they are applied to diabatic states. Will one give diabatic states that are more physical than the other?

\section{B. Extension to triplet-triplet Dexter transfer}

By maximizing Eq. (5), we create diabatic states that have localized charge density and, thus, are natural for electron transfer calculations. We now argue that with a small modification to Eq. (5), we can also construct diabatic states that will be appropriate for triplet-triplet Dexter energy transfer. ${ }^{45}$ Triplet-triplet transfer arises when an excited donor in a triplet state transfers its energy and spin to an acceptor which is originally in its ground singlet state: $D^{3 *} A^{1}$ $\rightarrow D^{1} A^{3 *}$. Thus, for triplet-triplet transfer, the diabatic states representing initial and final states must have spin density (rather than charge density) localized in different regions of space.

In order to produce diabatic states with localized spin density, we first decompose the dipole operator $\vec{\mu}$ as the sum of $\alpha$ and $\beta$ spin components:

$$
\begin{aligned}
\vec{\mu} & =\sum_{r, s=1}^{N_{b}} \vec{\mu}_{r s} a_{r \alpha}^{\dagger} a_{s \alpha}+\sum_{r, s=1}^{N_{b}} \vec{\mu}_{r s} a_{r \beta}^{\dagger} a_{s \beta} \\
& \equiv \vec{\mu}^{\alpha}+\vec{\mu}^{\beta} .
\end{aligned}
$$

Equation (40) is the usual second-quantized expansion for the dipole operator in a single particle basis of dimension $N_{b}: r$ and $s$ denote spinless single particle basis functions, $a^{\dagger}(a)$ signify creation (annihilation) operators, and the matrix elements are $\vec{\mu}_{r s}=\langle r|\vec{\mu}| s\rangle$. In Eq. (41), we define separate $\alpha$ and $\beta$ dipole operators according to the first and second terms on the right hand side of Eq. (40).

Finally, in order to localize spin density, we replace the localization sum in Eq. (5) by Eq. (42):

$$
\begin{aligned}
f_{\text {DexterTT }}(\mathbf{U})= & f_{\text {DexterTT }}\left(\left\{\Xi_{i}\right\}\right) \\
= & \sum_{i, j=1}^{N_{s}}\left|\left\langle\Xi_{i}\left|\vec{\mu}^{\alpha}\right| \Xi_{i}\right\rangle-\left\langle\Xi_{j}\left|\vec{\mu}^{\alpha}\right| \Xi_{j}\right\rangle\right|^{2} \\
& +\sum_{i, j=1}^{N_{s}}\left|\left\langle\Xi_{i}\left|\vec{\mu}^{\beta}\right| \Xi_{i}\right\rangle-\left\langle\Xi_{j}\left|\vec{\mu}^{\beta}\right| \Xi_{j}\right\rangle\right|^{2} .
\end{aligned}
$$

As in Eq. (5), $\Xi_{i}$ and $\Xi_{j}$ denote trial diabatic states which are to be determined by maximizing $f_{\text {DexterTT }}$, and $N_{s}$ is the total number of states. Equation (42) differs from Eq. (5) by ignoring the spin cross terms (i.e., $\vec{\mu}^{\alpha} \vec{\mu}^{\beta}$ terms) that would appear in expanding Eq. (5).

According to Eq. (42), we maximize the charge separation both between the spin-alpha charge centers and between the spin-beta charge centers. Thus, Eq. (42) should be a valid localization sum for Dexter triplet-triplet energy transfer. Moreover, like the standard Boys function [Eq. (5)], the DexterTT function [Eq. (42)] is also a quartic function of the rotation matrix and can be maximized using standard localization algorithms and without any computational penalty. ${ }^{35,41,46}$ The only computational difference between Eq. (5) and Eq. (42) is that, whereas maximization of the standard Boys function requires only the dipole matrix elements $\vec{\mu}_{i j}$ in the adiabatic basis, maximization of the DexterTT localization sum requires the separate spin-alpha and 


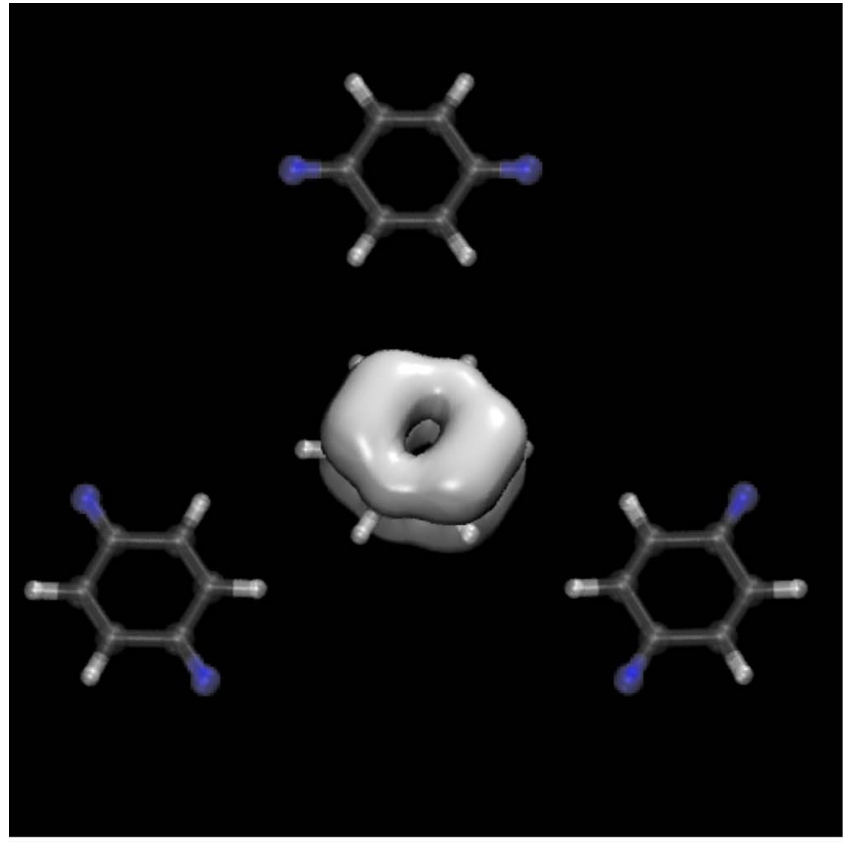

(a)

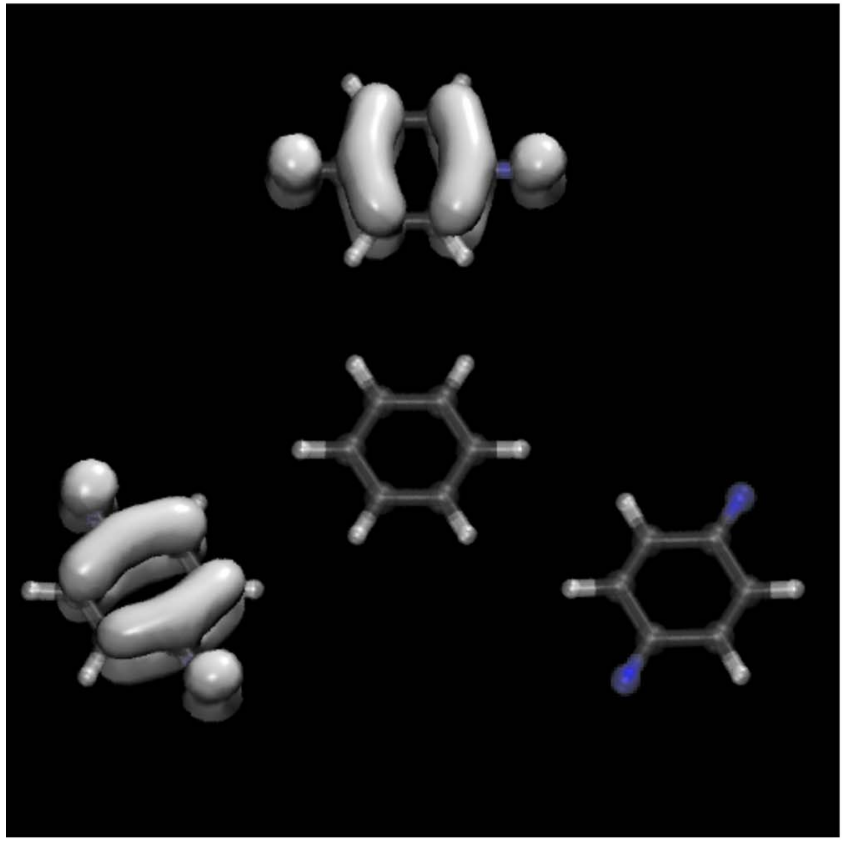

(b)

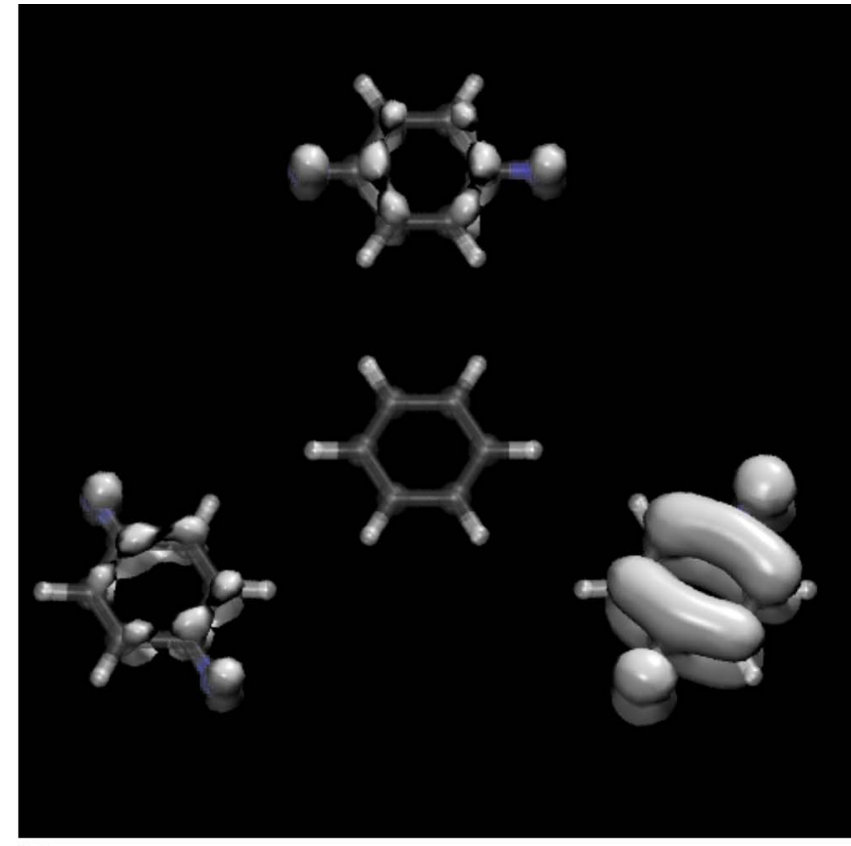

(c)

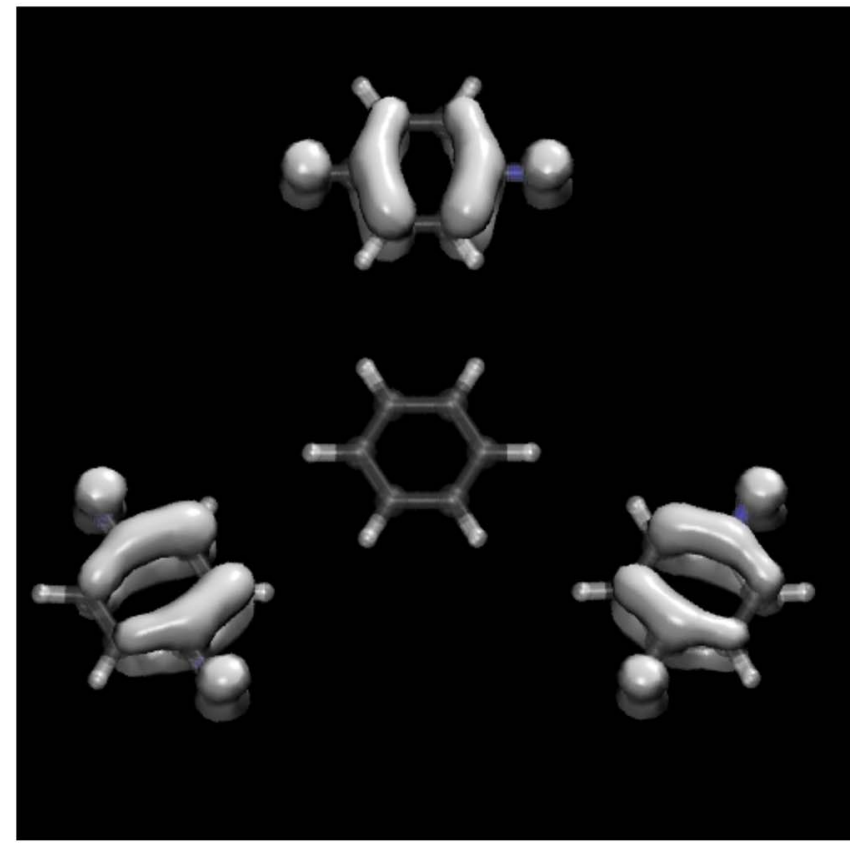

(d)

FIG. 1. (Color online) One detachment plot (a) and three attachment plots [(b)-(d)] for the adiabatic charge-transfer states. All detachment plots look similar to the eye, and thus, only one is included.

spin-beta dipole matrix elements in the adiabatic basis: $\vec{\mu}_{i j}^{\alpha}$, $\vec{\mu}_{i j}^{\beta}$. Diabatization according to Eq. (42) will be investigated in a future paper. ${ }^{42}$

\section{TWO CHEMICAL EXAMPLES FOR BOYS LOCALIZED STATES}

We now provide two simple examples for how one may use Boys localization to create diabatic states. Our first example is particular to excited state electron transfer, since the ground state of the model system will not be included in the diabatization scheme. Here, we consider benzene surrounded by three $p$-benzoquinone molecules, and we localize all ex- cited charge-transfer states. We do this in order to characterize qualitatively the Boys localized states using attachmentdetachment plots $^{47}$ (which are only meaningful when the ground state is unperturbed). Our second example is a square of four He atoms with one positive charge. Here, our Boys localization scheme mixes the ground-state of a molecular cluster with the first three excited states, and is more directly relevant to standard electron transfer theory.

\section{A. Benzene surrounded by $p$-benzoquinones}

Consider first the tetramer in Figs. 1 and 2. Here, benzene is surrounded by three $p$-benzoquinone molecules. Be- 


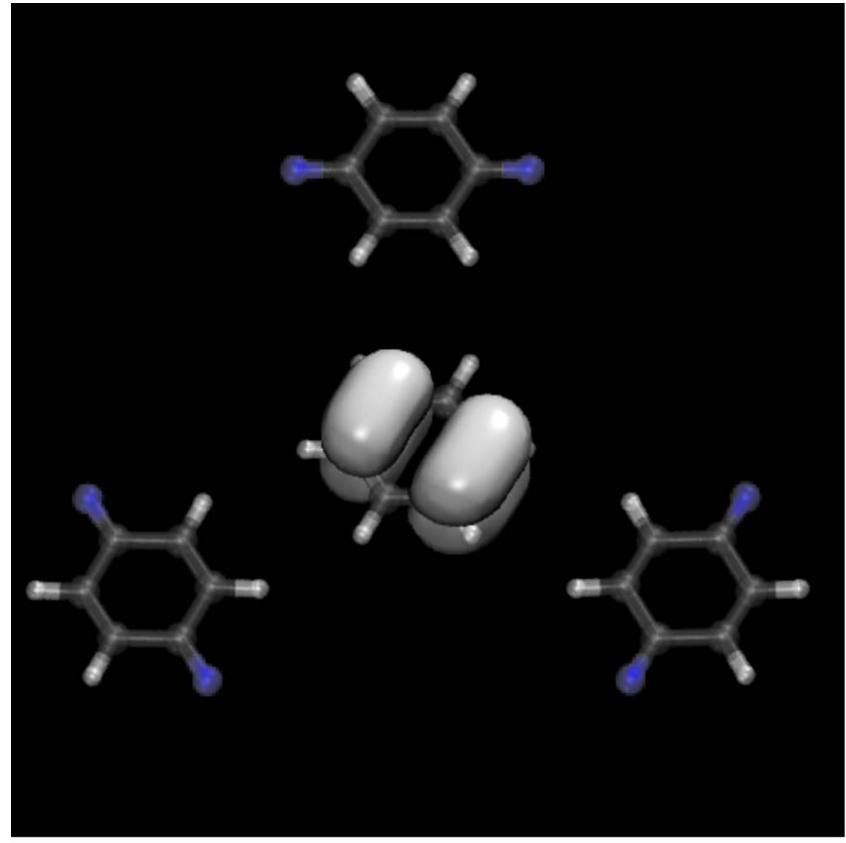

(a)

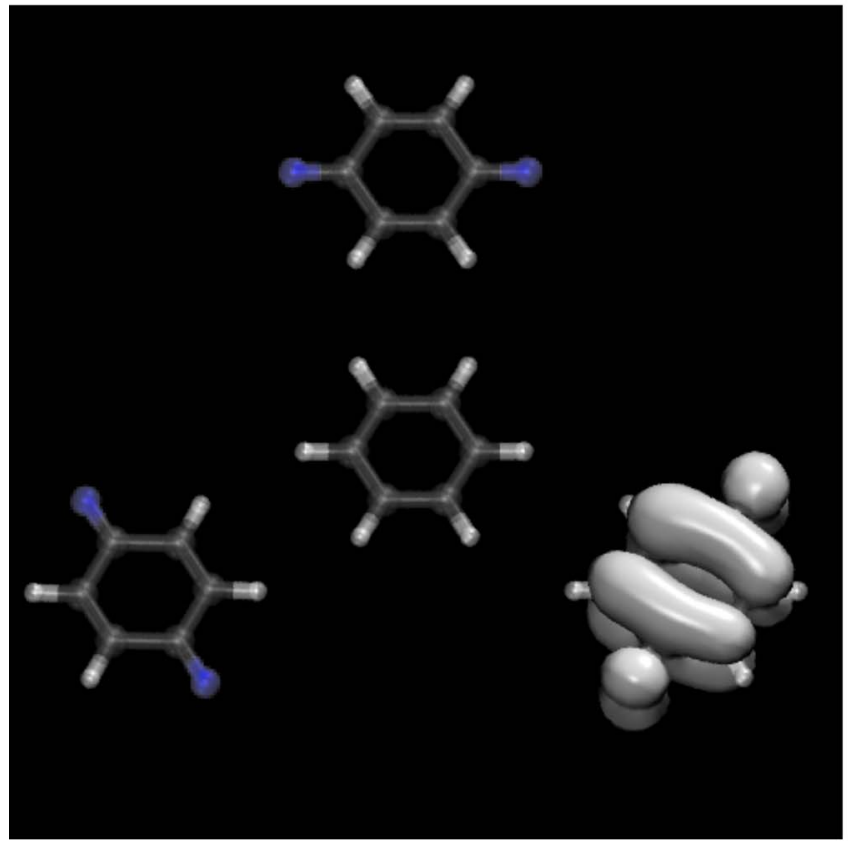

(b)

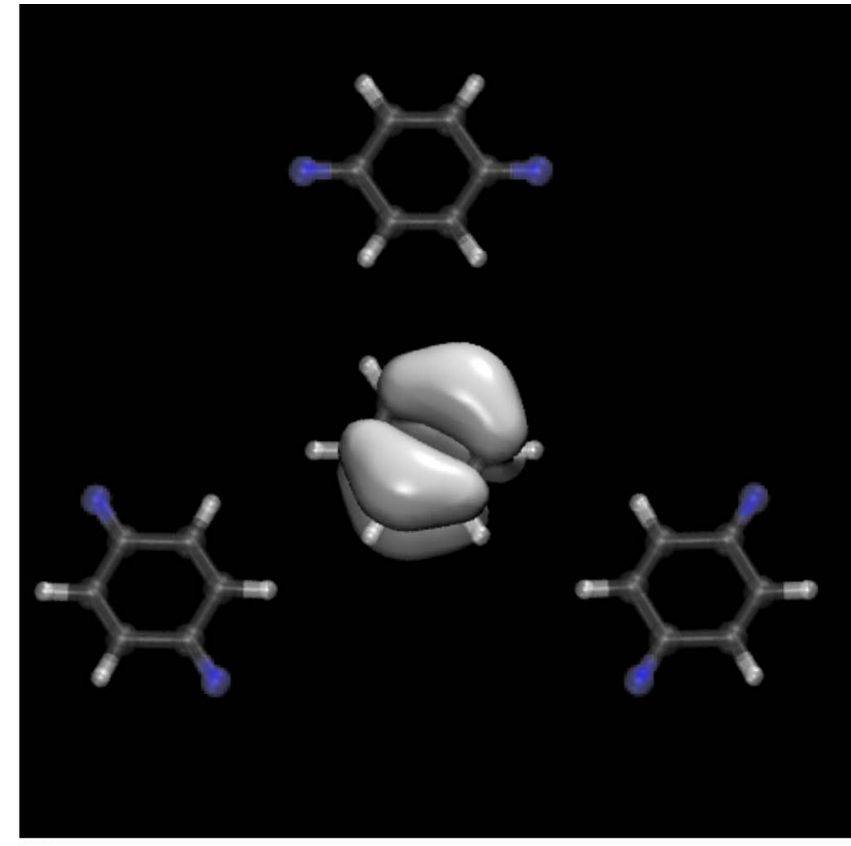

(c)

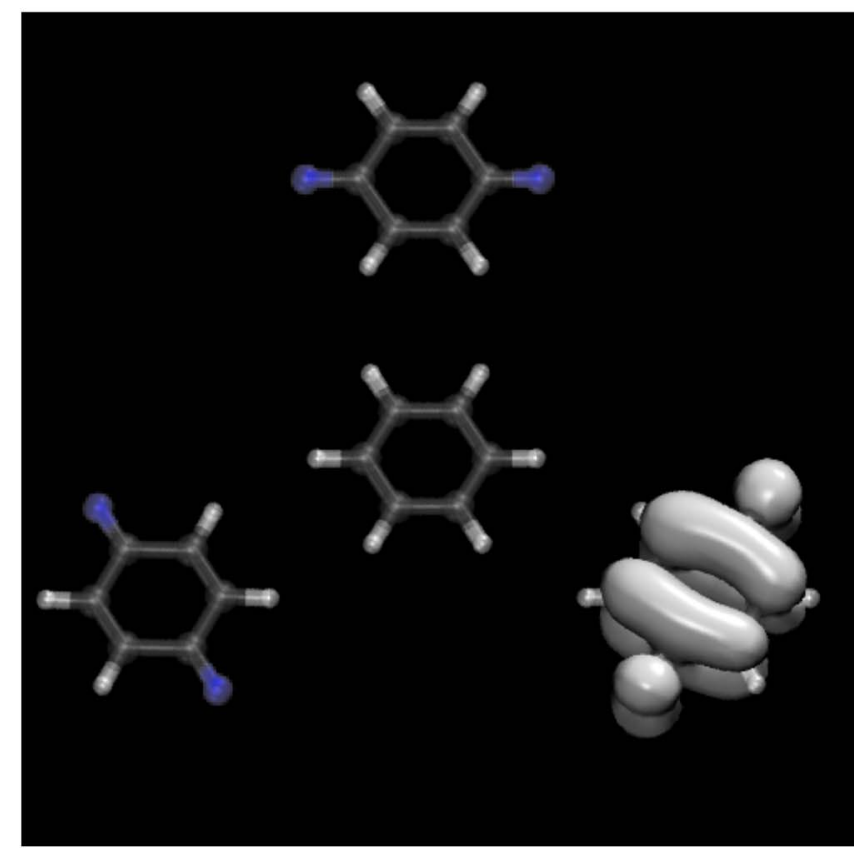

(d)

FIG. 2. (Color online) Detachment [(a) and (c)] and attachment plots [(b) and (d)] for two Boys localized diabatic states [(a)/(b) is one pair and (c)/(d) is another]. The attachment density in the other four states are simply rotated by $120^{\circ}$ and $-120^{\circ}$. The excitation energy for the state shown in (a)/(b) is 7.809 $\mathrm{eV}$ and for the state in (c)/(d) is $7.898 \mathrm{eV}$. These states differ by the orientation of the detached $\pi$ density on the benzene relative to the attached density on the quinone.

cause benzene is a good electron donor and $p$-benzoquinone is a good electron acceptor, we expect to find low-lying charge-transfer states for this molecular geometry. Indeed, a CIS calculation in a $6-31 G^{*}$ basis, reveals six low-lying charge-transfer states, in two groups of three isoenergetic states $(7.809$ and $7.897 \mathrm{eV})$. These charge-transfer states can be recognized by looking for large quadrupole moments of the one-electron attachment and detachment operators ${ }^{47}$ after a CIS calculation. One might expect that these adiabatic states should correspond to charge transfer from the highest occupied molecular orbital (HOMO) of benzene into the lowest unoccupied molecular orbital (LUMO) of $p$-benzoquinone. After all, the HOMO of benzene is doubly degenerate and we have a triply degenerate LUMO (with the LUMO's spread over the three acceptors), thus strongly suggesting that the six charge-transfer states are simply HOMOLUMO charge-transfer states.

In Fig. 1, we show three attachment and one detachment plot for the adiabatic charge-transfer states, noting that all other plots are duplicates. As described in detail in Ref. 47, these density plots are constructed by forming the oneelectron density matrices for an excited state $P_{\mathrm{ex}}$ and the 
ground state $P_{\mathrm{gs}}$, and then diagonalizing the difference matrix $P_{\text {diff }}=P_{\mathrm{ex}}-P_{\mathrm{gg}}$. By separating the positive and negative eigenvalues of $P_{\text {diff, }}$, one decomposes the difference density matrix into two new matrices, $P_{\text {diff }}=P_{\text {attach }}+P_{\text {detach }}$. The attachment density matrix has all non-negative eigenvalues and the electron density corresponding to $P_{\text {attach }}$ is an attachment plot; the corresponding detachment density matrix has all nonpositive eigenvalues.

From Fig. 1, one sees that, while the detached charge density visually appears as a linear combination of orbitals on the benzene, the attached charge density is spread out in these adiabatic states, as the benzene molecule may donate an electron into a linear combination of orbitals on the $p$-benzoquinones. The exact physical meaning of these attachment-detachment pictures is not entirely clear, however.

Next, using Q-CHEM, ${ }^{43}$ we perform a Boys localization on these charge-transfer states, and we find that the Jacobisweep algorithm converges in five steps, indicating a strong maximum. The algorithm correctly recognizes that it should separate these six charge-transfer states into three sets of two, forcing the dipole vectors to point into three different directions. In Fig. 2, we show attachment-detachment plots for two diabatic charge-transfer states after Boys localization. Now, the attachment plots for a diabatic state show charge density moved to one individual quinone molecule; the detachment plots show charge transferred from either of the two degenerate HOMO orbitals on the benzene. Because there are two different orientations for the detached $\pi$ density on the benzene relative to the attached density on the quinone, this difference in symmetry leads to a small difference in excitation energies (7.809 and $7.898 \mathrm{eV})$. The energies of the diabatic states are virtually unchanged from the adiabatic states because the coupling is so small, but there are nonzero mixings between the two sets of isoenergetic adiabatic states. The coupling between the two states in Fig. 2 (which have the same dipole moment but different attachment-detachment symmetry) is infinitesimal $(<4.0$ $\left.\times 10^{-7} \mathrm{eV}\right)$, as might be conjectured by symmetry. For states of the same symmetry but dipole moments at angles of $120^{\circ}$, the coupling is $2.6 \times 10^{-5} \mathrm{eV}$. For states of the opposite symmetry but dipole moments at angles of $120^{\circ}$, the coupling is $4.0 \times 10^{-6} \mathrm{eV}$.

The attachment-detachment plots for the other four diabatic excited states are identical to those in Fig. 2, only with the electronic density rotated by $120^{\circ}$ and $-120^{\circ}$. These plots make it clear that the doubly degenerate HOMO of the donor (benzene) is being transferred into the LUMO of individual acceptor $p$-benzoquinones, confirming our chemical intuition describing the charge transfer.

Interestingly, this model problem also suggests that Boys localization may be useful when trying to diabatize a conical intersection where there is charge transfer. Generating diabatic states in the vicinity of a conical intersection without calculating derivative couplings is an active area of current research. ${ }^{48}$

\section{B. $\mathrm{He}_{4}^{+}$}

Consider a square of four helium atoms with adjacent nuclei separated by $2 \AA$ and one positive charge on the cluster $\left(\mathrm{He}_{4}^{+}\right)$. CASSCF $(7,8)$ calculations have been performed on this cation using the GAMESS package ${ }^{49,50}$ in a $6-31 G^{*}$ basis. The energies of the ground state and first three adiabatic excited states are $-289.56644,-288.41488$, -288.41488 , and $-287.10486 \mathrm{eV}$, respectively. The energy of the fourth excited state is $35 \mathrm{eV}$ higher than the energy of the third excited state. Thus, the fourth excited state (and all higher excited states) has been disregarded.

The center of charge for each of the four included adiabatic states is the origin (i.e., the center of the $\mathrm{He}_{4}$-square) In other words, the positive charge (i.e., hole) is spread out over all four He atoms equally in all four states. If we consider a Huckel-type picture for the $\mathrm{He}_{4}^{+}$cation, the hole for the ground state will have no nodes, the hole for the (degenerate) first and second excited states will have one nodal line in the $\mathrm{He}_{4}$-plane, and the third excited state will have two nodal lines in the plane. Although we are working with a multiconfigurational expansion of the full wave function, this simple picture of the hole orbital can be confirmed by looking at dipole matrix elements between adiabatic states.

Now, when we perform Boys localization on the four adiabatic states (i.e., ground plus first three excited states) of the $\mathrm{He}_{4}^{+}$cluster, we find that the algorithm converges in five steps and the resulting diabatic states all have the same energy $(-288.37526 \mathrm{eV})$. Moreover, the charge centers for the four diabatic states make up a square with edge length of $1.96 \AA$, each one nearly on top of an atomic center. The edge length between diabatic states $(1.96 \AA)$ is slightly less than the edge length between He nuclei $(2 \AA)$ because the He nuclei are slightly stabilized by electron sharing.

For the $\mathrm{He}_{4}^{+}$cluster, the coupling matrix elements $\left(H_{A B}\right)$ are found to be $0.615 \mathrm{eV}$ between adjacent diabatic states and $0.040 \mathrm{eV}$ between diabatic states that are diagonally across from each other. This coupling matrix element should be compared to results for the dimer cation $\mathrm{He}_{2}^{+}$[where adiabatic states are generated with $\operatorname{CASSCF}(3,4)]$. For the dimer, at a radial distance of $2 \AA, H_{A B}$ is $0.617 \mathrm{eV}$, and for a distance of $2 \sqrt{2}=2.8284 \AA$, $H_{A B}$ is $0.082 \mathrm{eV}$. Thus, in $\mathrm{He}_{4}^{+}$, the $H_{A B}$ coupling element between adjacent He diabatic states is reduced by $0.002 \mathrm{eV}$, and the coupling element between diagonal diabatic states is decreased by $0.042 \mathrm{eV}$ (all relative to $\mathrm{He}_{2}^{+}$). The former difference is small but the latter difference is relatively large, demonstrating that diabatic states and the coupling elements between them can depend strongly on interactions from the environment, and emphasizing the need for an algorithm that can construct localized diabatic states from rigorously defined quantum-chemistry calculations.

Although we have picked two elementary problems for localizing states, the calculations above highlight the potential power of Boys localization, and its advantages over the standard GMH algorithm when constructing diabatic, localized charge-transfer states. The localized diabatic states above could not have been calculated using the GMH routine because, in these multicenter systems, there is no clear dipole moment arising from the original adiabatic states. Although 
it remains to thoroughly benchmark the Boys routine over a broad range of molecules, we expect the Boys algorithm presented here to greatly extend the applicability of the GMH algorithm, allowing one to diabatize adiabatic states from complicated noncollinear molecular geometries.

\section{CONCLUSIONS AND FUTURE WORK}

This paper has pointed out a straightforward connection between GMH and Boys localization. We have shown that the two are equivalent for two-state systems, and we have suggested that Boys localization should be applicable for constructing diabatic states when there are more than two noncollinear charge centers. We hope that this connection will be useful for chemists when modeling electron transfer in the future. Although Boys localization does not offer a simple formula for $H_{A B}$ as does the GMH formalism, the algorithm has already been implemented in most computational chemistry and physics programs in the context of orbital localization and should be easily transportable.

\section{ACKNOWLEDGMENTS}

We thank Ryan Steele and Anthony Dutoi for very helpful discussions and Mike Schmidt for help with the GAMESS program. J.E.S. was supported by a NSF International Research Fellowship. S.Y. was supported by a NDSEG fellowship. R.J.C. was pleased to acknowledge support from the Harvey Mudd College. M.A.R. thanks the chemistry division of NSF and ONR, and the DOD MURI program for support.

${ }^{1}$ F. T. Smith, Phys. Rev. 179, 111 (1969).

${ }^{2}$ T. F. O’Malley, Adv. At. Mol. Phys. 7, 223 (1971).

${ }^{3}$ M. Baer, Chem. Phys. Lett. 35, 112 (1975).

${ }^{4}$ C. A. Mead and D. G. Truhlar, J. Chem. Phys. 77, 6090 (1982).

${ }^{5} \mathrm{H}$. Koppel, in Conical Intersections: Electronic Structure, Dynamics and Spectroscopy, edited by W. Domcke, D. R. Yarkony, and H. Koppel (World Scientific, New Jersey, 2004), p. 175.

${ }^{6}$ L. S. Cederbaum, in Conical Intersections: Eletronic Structure, Dynamics and Spectroscopy, edited by W. Domcke, D. R. Yarkony, and H. Koppel (World Scientific, New Jersey, 2004), p. 3.

${ }^{7}$ M. Baer, Beyond Born-Oppenheimer: Electronic Nonadiabatic Coupling Terms and Conical Intersections (Wiley, New York, 2006).

${ }^{8}$ Z. H. Top and M. Baer, J. Chem. Phys. 66, 1363 (1977).

${ }^{9}$ M. Baer, Mol. Phys. 40, 1011 (1980).

${ }^{10}$ G. J. Halasz, A. Vibok, S. Suhai, and M. Baer, J. Chem. Phys. 127, 244101 (2007).

${ }^{11}$ T. Pacher, L. S. Cederbaum, and H. Koppel, J. Chem. Phys. 89, 7367 (1988).

${ }^{12}$ T. Pacher, L. S. Cederbaum, and H. Koppel, Adv. Chem. Phys. 84, 293 (1993).

${ }^{13}$ H. Nakamura and D. G. Truhlar, J. Chem. Phys. 115, 10353 (2001).

${ }^{14}$ H. Nakamura and D. G. Truhlar, J. Chem. Phys. 117, 5576 (2002).

${ }^{15}$ H. Nakamura and D. G. Truhlar, J. Chem. Phys. 118, 6816 (2003).

${ }^{16}$ K. Ruedenberg and G. J. Atchity, J. Chem. Phys. 99, 3799 (1993).

${ }^{17}$ G. J. Atchity and K. Ruedenberg, Theor. Chem. Acc. 97, 47 (1997).

${ }^{18}$ M. Pavlovic, J. Kucar, and J. Hendekovic, Int. J. Quantum Chem. 32, 705 (1987).

${ }^{19}$ W. Domcke and C. Woywood, Chem. Phys. Lett. 216, 362 (1993).

${ }^{20}$ W. Domcke, C. Woywood, and M. Stengle, Chem. Phys. Lett. 226, 257 (1994).

${ }^{21}$ R. J. Cave and M. D. Newton, Chem. Phys. Lett. 249, 15 (1996).

${ }^{22}$ R. J. Cave and M. D. Newton, J. Chem. Phys. 106, 9213 (1997).

${ }^{23}$ There is also the question of how big are the derivative couplings arising from the GMH method, and we are unaware of any computational studies estimating them. Conversely, however, there is an analytical expression by Kryachko, specific to the two-state problem, showing how big the off-diagonal dipole element ought to be (rather than zero, which is the GMH ansatz) in order for the derivative couplings to vanish. See Refs. 51 and 52.

${ }^{24}$ H. M. McConnell, J. Chem. Phys. 35, 508 (1961).

${ }^{25}$ J. N. Onuchic and D. N. Beratan, J. Chem. Phys. 92, 722 (1990).

${ }^{26}$ I. V. Kurnikov and D. N. Beratan, J. Chem. Phys. 105, 9561 (1996).

${ }^{27}$ D. N. Beratan and S. S. Skourtis, Curr. Opin. Chem. Biol. 2, 235 (1998).

${ }^{28}$ A. A. Stuchebrukhov, Chem. Phys. Lett. 225, 55 (1994).

${ }^{29}$ A. A. Stuchebrukhov, Adv. Chem. Phys. 118, 1 (2001).

${ }^{30}$ S. Larsson and L. Rodriguez-Monge, J. Photochem. Photobiol., A 82, 61 (1994).

${ }^{31}$ C. P. Hsu and R. A. Marcus, J. Chem. Phys. 106, 584 (1997).

${ }^{32}$ J. E. Lennard-Jones and J. A. Pople, Proc. R. Soc. London, Ser. A 202, 166 (1950).

${ }^{33}$ S. Saebo and P. Pulay, Annu. Rev. Phys. Chem. 44, 213 (1993).

${ }^{34}$ J. M. Foster and S. F. Boys, Rev. Mod. Phys. 32, 300 (1960).

${ }^{35}$ C. Edmiston and K. Ruedenberg, Rev. Mod. Phys. 35, 457 (1963).

${ }^{36}$ S. F. Boys, in Quantum Theory of Atoms, Molecules and the Solid State, edited by P. Lowdin (Academic, New York, 1966), p. 253.

${ }^{37}$ J. Pipek and P. G. Mezey, J. Chem. Phys. 90, 4916 (1989).

${ }^{38}$ D. A. Kleier, T. A. Halgren, J. H. Hall, and W. N. Lipscomb, J. Chem. Phys. 61, 3905 (1974).

${ }^{39}$ Confusing as it must sound, credit for the "Boys" orbitals should actually be attributed both to Edmiston and Ruedenberg, as well as Foster and Boys. Foster and Boys suggested in 1960 (Ref. 34) the criterion of maximizing the (nonlinear) product of the distances between orbital centroids, while Edmiston and Ruedenberg improved on this criterion in 1963 (Ref. 35 ), proposing to maximize the (linear) sum of the distances between orbital centroids. The latter definition is the criterion used today for "Boys" orbitals. Furthermore, Edmiston and Ruedenberg were the first to suggest a formal technique for computing these orbitals exactly (rather than approximately, as Foster and Boys had proposed).

${ }^{40}$ This derivative can be done without too much difficulty by following the analogous calculations in Refs. 35 and 41.

${ }^{41}$ J. E. Subotnik, Y. Shao, W. Liang, and M. Head-Gordon, J. Chem. Phys. 121, 9220 (2004).

${ }^{42}$ S. Yeganeh, R. J. Cave, M. A. Ratner, and J. E. Subotnik (unpublished).

${ }^{43}$ Y. Shao, L. Fusti-Molnar, Y. Jung, J. Kussmann, C. Ochsenfeld, S. T. Brown, A. T. B. Gilbert, L. V. Slipchenko, S. V. Levchenko, D. P. O’Neill, R. A. Distasio, R. C. Lochan, T. Wang, G. J. O. Beran, N. A. Besley, J. M. Herbert, C. Y. Lin, T. Van Voorhis, S. H. Chien, A. Sodt, R. P. Steele, V. A. Rassolov, P. E. Maslen, P. P. Korambath, R. D. Adamson, B. Austin, J. Baker, E. F. C. Byrd, H. Dachsel, R. J. Doerksen, A Dreuw, B. D. Dunietz, A. D. Dutoi, T. R. Furlani, S. R. Gwaltney, A. Heyden, S. Hirata, C.-P. Hsu, G. Kedziora, R. Z. Khalliulin, P. Klunzinger, A. M. Lee, M. S. Lee, W. Liang, I. Lotan, N. Nair, B. Peters, E. I. Proynov, P. A. Pieniazek, Y. M. Rhee, J. Ritchie, E. Rosta, C. D. Sherrill, A. C. Simmonett, J. E. Subotnik, H. L. Woodcock III, W. Zhang, A. T. Bell, A. K. Chakraborty, D. M. Chipman, F. J. Keil, A. Warshel, W. J. Hehre, H. F. Schaefer, J. Kong, A. I. Krylov, P. M. W. Gill, and M. Head-Gordon, Phys. Chem. Chem. Phys. 8, 3172 (2006).

${ }^{44}$ If we apply the Pipek-Mezey approach to states, rather than orbitals, the resulting algorithm will construct diabatic states by maximizing the sum of the squares of atomic populations. Notably, construction of diabatic states by maximizing the squares of natural spin-orbital populations was suggested 20 years ago in Ref. 18. These two approaches are different, however, because by maximizing the squares of atomic (rather than orbital) populations, the PM algorithm forces localization of the diabatic states; there is no need for such localization in the approach of Ref. 18.

${ }^{45}$ Z. Q. You, C. P. Hsu, and G. Fleming, J. Chem. Phys. 124, 044506 (2006).

${ }^{46}$ J. E. Subotnik and M. Head-Gordon, J. Chem. Phys. 122, 034109 (2005).

${ }^{47}$ M. Head-Gordon, A. M. Grana, D. Maurice, and C. A. White, J. Phys. Chem. 99, 14261 (1995).

${ }^{48}$ I. Ryb and R. Baer, J. Chem. Phys. 121, 10370 (2004).

${ }^{49}$ M. W. Schmidt, K. K. Baldridge, J. A. Boatz, S. T. Elbert, M. S. Gordon, J. H. Jensen, S. Koseki, N. Matsunaga, K. A. Nguyen, S. Su, T. L. 
Windus, M. Dupuis, and J. A. Montgomery, J. Comput. Chem. 14, 1347 (1993).

${ }^{50}$ M. S. Gordon and M. W. Schmidt, in Theory and Applications of Computational Chemistry: The First Forty Years, edited by C. E. Dykstra, G.
Frenking, K. S. Kim, and G. E. Scuseria (Elsevier, Amsterdam, 2005), p. 1167.

${ }^{51}$ E. S. Kryachko, J. Phys. Chem. A 103, 4368 (1999).

${ }^{52}$ E. S. Kryachko, Adv. Quantum Chem. 44, 119 (2003). 